\title{
El molino de marea del Río Arillo Un patrimonio por recuperar
}

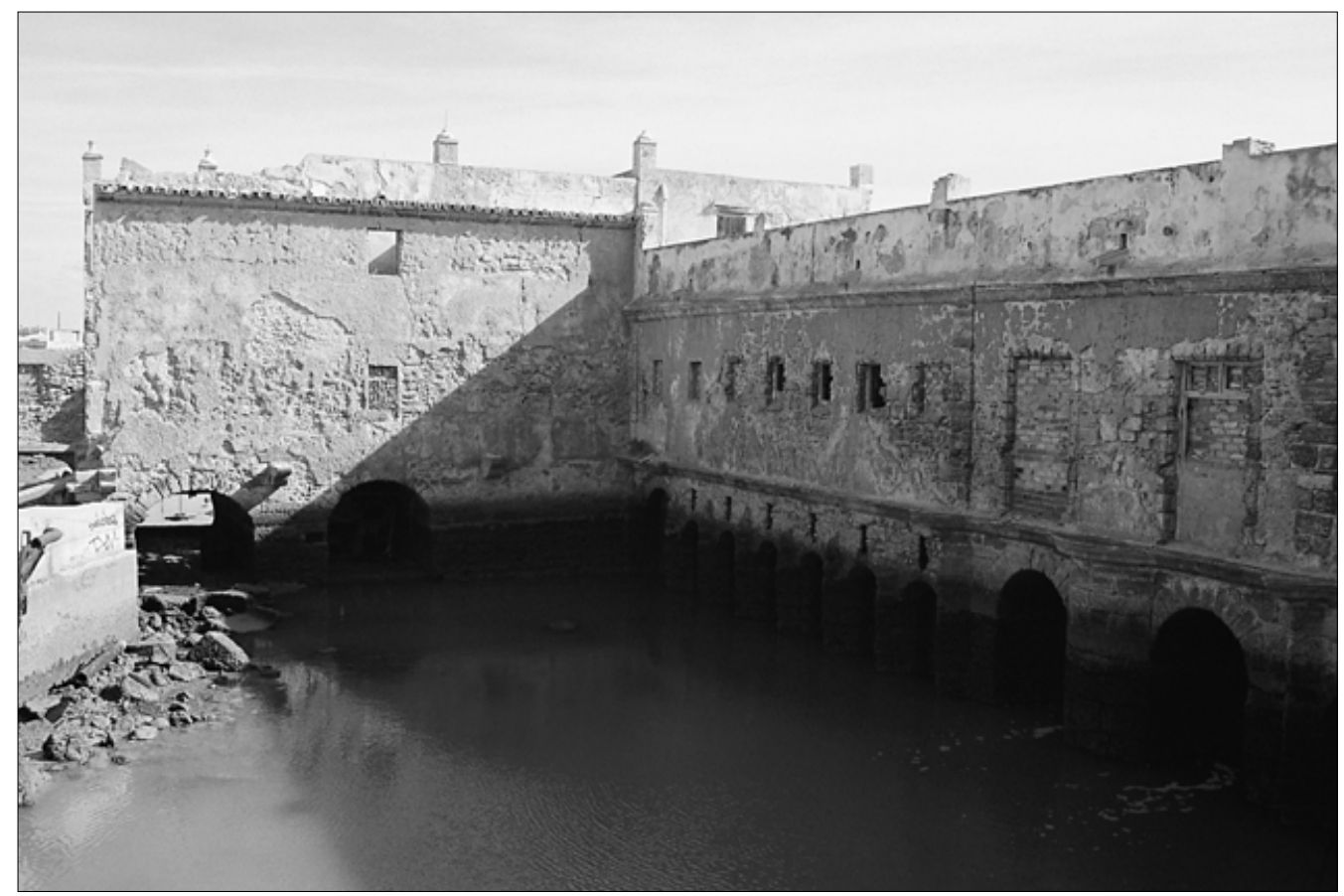

Vista actual de la fachada principal del molino.

José Ramón Barros Caneda Historiador del Arte

\section{Antonio Tejedor Cabrera}

Arquitecto

La bahía gaditana, tanto por sus características morfológicas como económicas, fue un marco geográfico que favoreció la creación a lo largo de lo siglos de un elevado número de molinos de marea. Marismas, caños y ríos vieron instalarse en sus márgenes estos edificios preindustriales que aprovechaban la dinámica de las mareas como fuerza motriz de sus instalaciones. Desde el siglo XVI, existieron hasta 17 molinos, siete de ellos alrededor de la Isla de León, según ha señalado Loic Menanteau '; tal fue la importancia de estas arquitecturas productivas cuyo esquema tipológico fue recogido por destacados tratadistas de la arquitectura hidráulica como Belidor.

Las primeras noticias históricas nos trasladan al período comprendido entre 1547 y I 585 en el que Francisco López realizó el dibujo de un molino de esta naturaleza ubicado en Puerto Real ${ }^{2}$. Pero fue durante los siglos XVII y XVIII cuando los molinos proliferan en esta zona gracias a la vinculación con las salinas y al aumento de la demanda de cereales producida como consecuencia de la intensa relación económica con América a través del comercio marítimo y el consiguiente aumento demográfico. El siglo XIX continuará con la fase de expansión de los molinos que, durante el primer cuarto de siglo, siguen usando las técnicas tradicionales para progresivamente ir sustituyendo las fuerzas naturales por el vapor y posteriormente por la electricidad hasta caer en desuso, lo que llevó a un progresivo abandono y posterior ruina o destrucción ${ }^{3}$. 
$\longleftarrow$
늠

Fig. I. Planta del primitivo proyecto del molino del Río Arillo. 1798. Pedro de Vega.

Fig. 2. Plano del terreno concedido a Miguel

Álvarez para la construcción del molino de Río Arillo.

Julián Albo y Helguero. 1798.
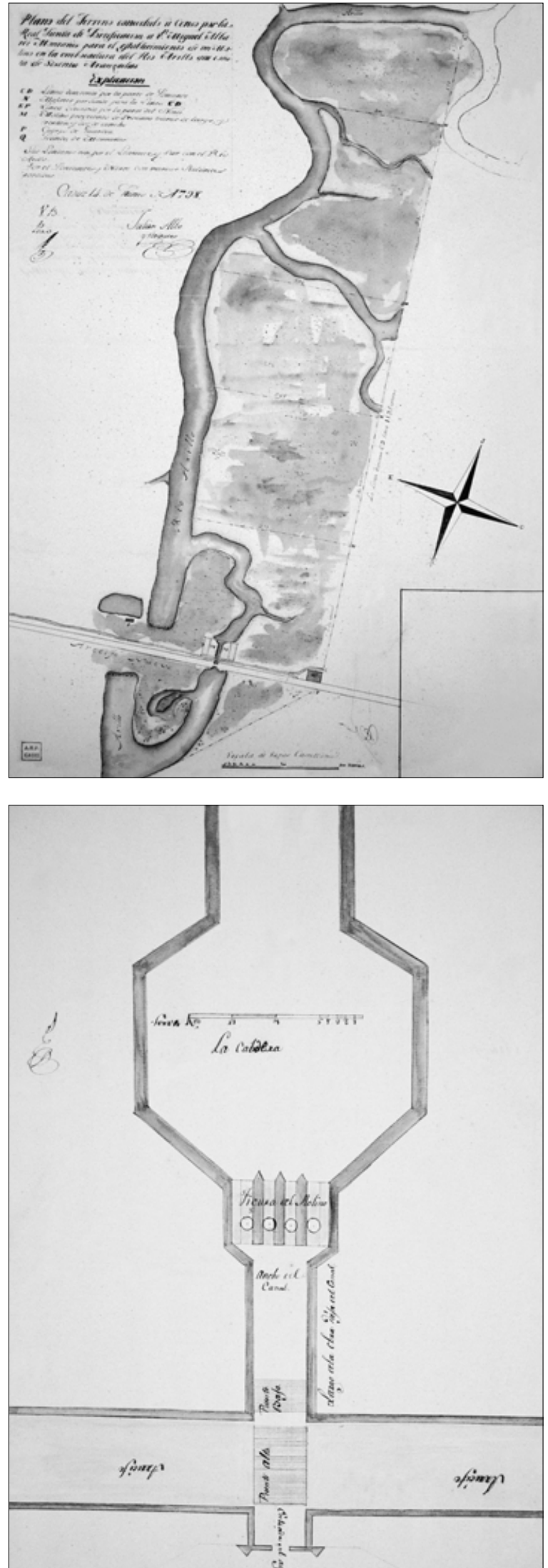

Dentro de estos años previos a la Revolución Industrial española se localiza este molino de mareas que se nombra del Río Arillo ${ }^{4}$, y que se construirá en el caño del mismo nombre, en el límite del término municipal de Cádiz. La realización en 1773 de un pa- drón de quintas nos permite conocer la situación de la zona en los años previos a la construcción. Pilar Ruiz la define como un ámbito muy disperso, de carácter rural y salinero, en el que el Río Arillo figuraba como frontera natural entre los términos de Cádiz y San Fernando y cuyos referentes demográficos y arquitectónicos lo constituían una "tienda de montañés", dos molinos Ilamados de Santibáñez y el Molino del Fraile, y dos salinas en activo ${ }^{5}$.

Un factor de influencia decisiva en la consideración del terreno para la construcción del molino fue el nuevo trazado del arrecife o Camino Real desde Cádiz a la Isla de León. Una industria de esta naturaleza enfocada a la elaboración de harina para suministro a las ciudades y a las importantes actividades marítimas de la zona, bien civiles, bien militares, debía contar con una infraestructura de comunicación adecuada. El primitivo trazado del camino a San Fernando no giraba en Torregorda, sino que enlazaba con la zona actual de Camposoto ${ }^{6}$. Será a partir del terremoto de 1755, que le afectó de manera importante, cuando se iniciarán las reparaciones y la modificación del trazado de la carretera, que se consolidó definitivamente en 17897. De esta manera, el nuevo trazado se convertía en eje fundamental del transporte de mercancías, al margen de que también el propio río Arillo fuera una esencial vía de comunicación con la bahía gaditana. La elección del lugar donde construir el molino quedaba así definida por las condiciones orográficas e hidráulicas que permitían tal tipo de actividad industrial y, a la vez, por la existencia de las adecuadas infraestructuras. De hecho, veremos que el camino y el puente, ambos de crucial valor estratégico, serán un motivo constante de conflictos y su presencia generará unas condiciones especiales en el régimen de explotación de este terreno.

En esta situación de reordenación y transformación, Miguel Álvarez Montañés ${ }^{8}$ solicita a la Real Junta de Fortificaciones en marzo de 1798 la concesión a censo perpetuo de un terreno situado en la desembocadura del Río Arillo para construir un molino de harina de "quatro o seis piedras" 9 . El escrito que dirige al presidente de la Junta de Fortificaciones identifica con claridad las necesidades de índole social y económica de la zona, que serán esgrimidas como poderosos argumentos para que la petición fuera tomada en consideración. En primer lugar, Miguel Álvarez menciona el aumento del abastecimiento de harina protegiéndose la zona contra posibles carestías que se dieron en otras épocas, asegurando de esta manera el suministro "de la elaboración del trigo para su vecindario, tropa de guarnición, esquadra y Arcenal". Además, manejando conceptos de teoría económica, se refiere a la incidencia que este aumento de producción tendría en los precios, lo que ciertamente supondría un importante beneficio.

En segundo lugar, pretende hacer productiva una zona inculta de marismas y, en principio, sin valor por cuanto quedaba inutilizada al anegarse por las mareas, construyendo un edificio que crearía no só- 
lo el empleo y la actividad propios de la construcción, sino también el trabajo que generaría el funcionamiento y mantenimiento del molino. Su presencia, por otra parte, apoyaría el asentamiento de población que junto con la tienda que quedaba comprometido a crear, haría que la zona estuviera más habitada y en consecuencia vigilada, suprimiéndose de esa manera el retén militar existente. Además, se comprometía a la reparación y mantenimiento permanente del puente sobre el Río Arillo, que en ese momento estaba casi en ruina, librando de ese gasto a la Junta de Fortificaciones. En suma, las ventajas que traería la construcción del molino podrían resumirse en tres palabras que utiliza Miguel Álvarez al principio de su memorial y que son compendio de toda una ideología: utilidad, conveniencia y beneficio, en las que subyace la idea de obtener grandes ventajas públicas a través de la promoción de la actividad económica ${ }^{10}$

El proyecto pasó a informe del ingeniero director, por aquel entonces Luis Huet, el cual el 18 de marzo lo valoró positivamente al tomar en consideración sus características y no presentar problemas de seguridad militar por estar situado a una legua de la ciudad de Cádiz y no contravenir así las Reales Órdenes de Fortificaciones. El cambio de destino de Huet obligó a su sustituto, José del Pozo 'I, a elaborar un nuevo informe donde establece las condiciones definitivas para escriturar el censo. A la vez encarga a otro ingeniero, Julián Albo y Helguero 12, realizar las mediciones y levantar el plano de los terrenos cedidos, estableciéndose una superficie de 384.000 varas cuadradas equivalentes a 60 aranzadas. Con respecto al censo que se debía pagar y, tomando como referencia lo que se tributaba por los terrenos de huertas del frente de Puerta de Tierra, se establece un costo censal de 33 reales de vellón anuales por aranzada. A ello se añade una cantidad de 30 reales al mes por los derechos de pesca.

Realizados los trámites y comunicaciones oportunas, el 16 de julio de 1798 se otorgan las escrituras del censo de las citadas tierras. En ellas se establecen los límites del terreno que se define como "de 60 aranzadas, situado en la desembocadura del río Arillo en el término de Cádiz y que linda por el levante y el sur con el propio río y por el poniente y el norte con salinas en desuso", así como las condiciones de los censos abonables y algunos acuerdos especiales. En primer lugar se pacta la reconstrucción y mantenimiento a cargo del solicitante del puente alto y bajo sobre el río ${ }^{13}$. Se incluye la conservación del edificio del molino y del almacén de harina y la prohibición de realizar labores de pesca que entorpezcan la actividad ${ }^{14}$. Además, debía pagarse, pese a ser un edificio y una actividad cuyos rendimientos iban a ser elevados, una contribución de 360 reales de vellón al año correspondiente a la cuota que pagaban las tiendas situadas fuera de la muralla gaditana. Esta contribución, como decimos menor, se reducía en honor a las aportaciones y obligaciones de gasto que el interesado contraía. Finalmente, se acordaba también no impedir ni obstaculizar el trán- sito por el puente, derribar el edificio si el interés militar lo exigía, sin esperar compensación alguna y que el terreno y todo lo en él construido era indivisible e intransferible.

Bajo estas condiciones se edificó el molino y con tales condiciones comenzó a funcionar. Sin embargo, la lindera carretera de Cádiz a San Fernando, así como el propio recorrido del río van a suponer un serio problema para su funcionamiento, que se verá afectado a los pocos años de iniciar la actividad. En 1810 se planteó la posibilidad de establecer una conexión entre la bahía y el mar haciendo navegable el río. De esta manera se iniciaron las obras abriéndose un canal y construyéndose un nuevo puente en el camino de Cádiz a San Fernando un poco más arriba del molino. Esta obra dio lugar a que el agua del río se canalizara por esta nueva apertura dejando fuera del recorrido al molino. La situación paralizó la

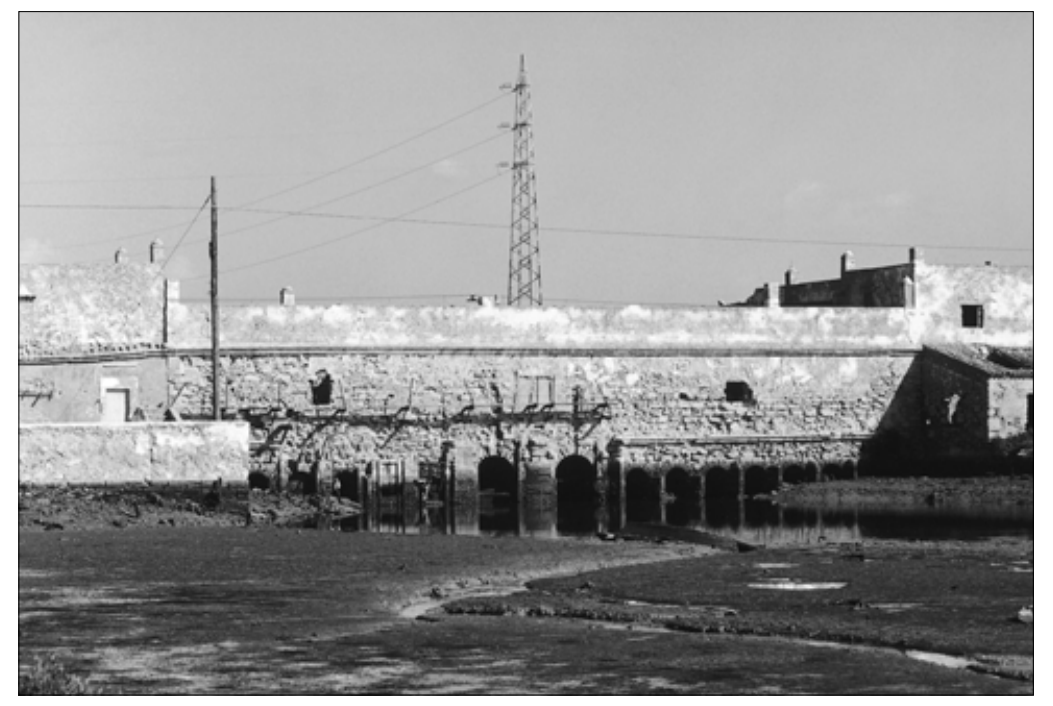

Vista posterior del molino.

actividad del inmueble y obligó a realizar obras para adaptarlo al nuevo cauce. De estos años puede ser la crujía lateral con los dos arcos de medio punto labrados en cantería, cuya función era devolver el agua, una vez utilizada, al nuevo trayecto.

De cualquier forma, parece ser que el molino quedó paralizado, lo que afectó a su estado de conservación, dejándose de pagar, en consecuencia, los censos y demás tributos y aportaciones concertados en la escritura. En este estado de cosas pasó la propiedad del edificio a Juan Dámaso López que obtiene la redención de la deuda que ascendía en el año | 82 | a 29.700 reales, y la liberación del censo que se impuso en 1798. A cambio, Juan Dámaso se comprometía a cegar el puente del inacabado proyecto de 1810, ya inservible, y recomponer el camino en ese tramo con lo cual el río retomaría su primitivo curso, asegurándose de nuevo el funcionamiento del molino, que ahora vería aumentada su potencia al concedérsele una ampliación del número de arcos de la crujía lateral. A estas fechas 
$\longleftarrow$
$\square$ por tanto debe corresponder el tramo de los dos arcos escarzanos construidos en ladrillo de la crujía lateral del molino, así como la parte del edificio construida sobre ellos. Finalmente, y en este mismo proceso de recuperación de la actividad, el nuevo propietario recibía autorización para ampliar la luz del arco del primitivo puente a través del cual recibía el agua, comprometiéndose, según constaba en la escritura original, al mantenimiento y buen uso del citado puente. En 1826, los ingenieros militares franceses hacen un levantamiento del alzado del molino en el que puede apreciarse la situación del mismo y en el que aparece el ala lateral, así como el balcón del cuerpo principal y un remate en forma de cruz coronándolo ${ }^{15}$.

En 1836, tras el fallecimiento de Juan Dámaso López, el molino pasó a propiedad de los hermanos García Lizarza, Juan y Félix, que tras escritura de transacción otorgada en 1847 quedó definitivamente en manos de Félix García Lizarza aunque hipotecado como aval para cubrir la parte de la herencia que correspondía a su hermano ${ }^{16}$. Finalmente pasó a formar parte de diversas herencias hasta que fue vendido en 1977, ya totalmente desmantelado, a la empresa Unión Salinera de España, S.A. su actual propietaria 17.

El proceso histórico del molino nos ha permitido interpretar y conocer las etapas constructivas del edificio y los cambios que en su estructura y funcionamiento se han dado. En este sentido podemos distinguir una fase de proyecto y dos constructivas, quedando las dos primeras muy relacionadas y la tercera como conformadora de la actual fisonomía del inmueble.

Cuando en 1798 Miguel Álvarez solicitó a la Junta de Fortificaciones los terrenos acompañó a su memorial una planta esquemática del proyecto que tenía intención de ejecutar. Esta planta mostraba y dibujaba el molino formado por un canal, que era el lugar por el que el río salvaba el arrecife a San Fernando y que comunicaba con el inmueble, como hemos visto proyectado inicialmente para cuatro

Vista actual de la crujía lateral.

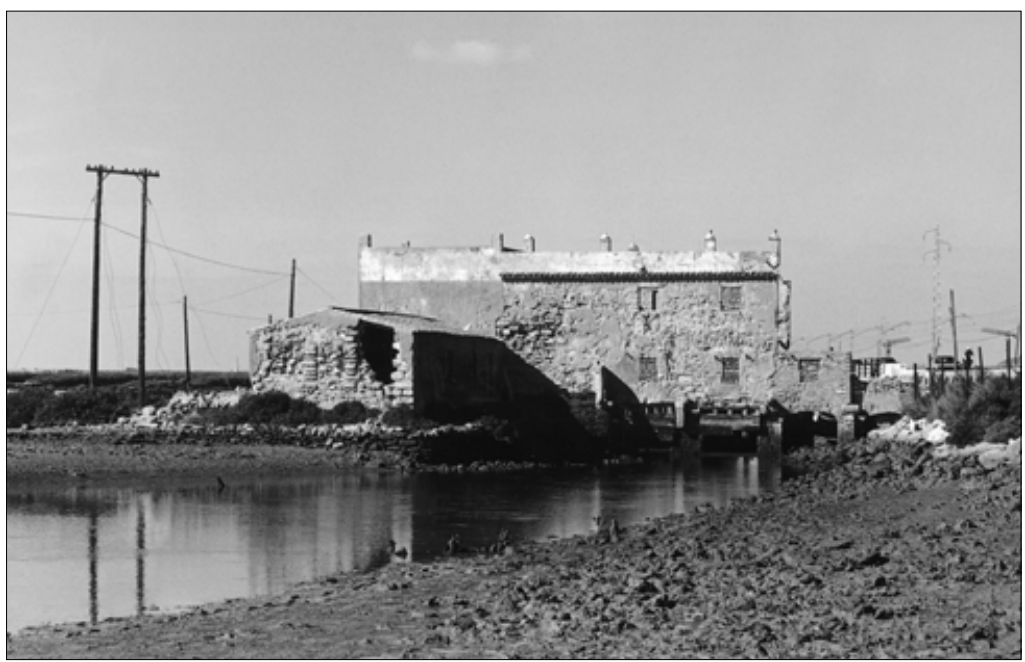

piedras que son las que aparecen reflejadas en la planta, y con dos canales de desagüe situados en los extremos del edificio. A continuación se abría, con planta octogonal, la caldera a la que seguía la canalización del río. En este proyecto inicial que corresponde con el verdadero sistema de funcionamiento del molino en relación con la trayectoria del agua, la caldera se encontraba situada en la parte posterior (aún hoy día pueden verse restos de la misma formados por hileras de cantería de piedra ostionera). El funcionamiento en este proyecto se basaba en el flujo y reflujo de la marea. Cuando la marea subía el agua entraba por el canal y, atravesando las compuertas de los extremos del molino, permitía que la marea subiese en todo el caño. Con la bajamar, las compuertas basculantes, que antes habían facilitado el pase del agua, se bloqueaban y el agua del reflujo se canalizaba a través de las compuertas de salida a los rodeznos del molino, desaguando de nuevo al canal desde donde fluía al cauce del río.

Éste era, como decimos, el sistema idóneo y acorde con el sentido del fluir de las aguas del caño, y así debió construirse el molino inicial al cual corresponde la crujía mayor que parece se ejecutó con trece piedras ${ }^{18}$. Sin embargo, hemos visto que en I 810 las nuevas obras de la carretera unieron el brazo mayor del río originando deficiencias en el flujo de agua. A este momento corresponde la tercera y última fase constructiva y las modificaciones en la configuración y recorrido del caudal del agua en el molino. La modificación esencial consistió en crear dos canales laterales. De esta manera, con el flujo o la crecida se bloqueaban las compuertas basculantes de estos dos canales nuevos, y con el reflujo el agua, tras mover los rodeznos, se evacuaría al nuevo cauce, por las compuertas laterales, hacia el río que fluía en bajamar en dirección a la bahía. Este sistema fue ampliado en dos canales más, volviendo a retomarse el procedimiento original al cegarse el puente nuevo y volver el río a su curso primitivo.

El edificio presenta una composición esquemática, funcional y de gran rigor geométrico. Estos tres rasgos están muy relacionados con arquitecturas de tipología industrial y militar que se vinculan en la zona a la labor de los ingenieros militares o al menos a la influencia que su labor dejó. Las tres características facilitan la construcción de un edificio útil, económico y fácilmente extensible al podérsele acoplar, como de hecho sucedió, nuevas estructuras que complementaran o modificaran las existentes.

Nada sabemos del arquitecto o ingeniero que realizó la primera obra del molino. Puede suponerse que el autor material fuese el mismo Pedro de Vega que firmó el proyecto 19. Lo que sí está claro es que el autor, fuera éste u otro, era conocedor de la tratadística arquitectónica y de las fórmulas estilísticas que se desarrollaban en las ciudades del entorno. Desde este punto de vista, resulta sorprendente la similitud que la planta primitiva del proyecto pre- 


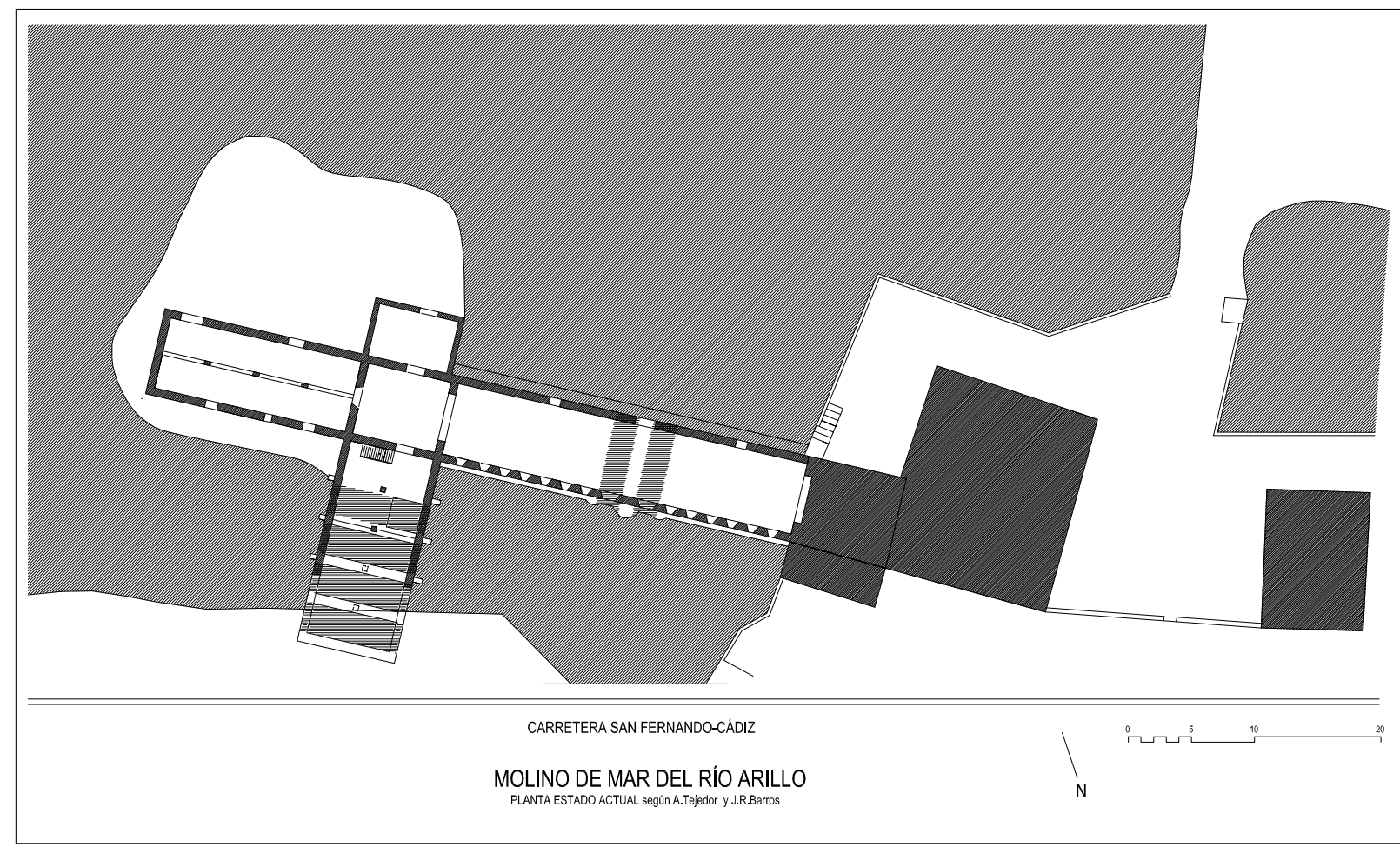

senta con la reproducción que el tratadista boloñés Serlio hace del puerto de Ostia, de la que toma el esquema general y más concretamente la parte del canal de entrada y el ensanche que da acceso al interior del puerto ${ }^{20}$. Del mismo modo hay que hacer notar ciertas similitudes entre los modelos de puentes recogidos por el citado Serlio y los arcos de la parte inferior del inmueble en los que destaca el uso de tajamares apuntados y redondeados ${ }^{21}$.

Pero si el autor era buen conocedor de la tratadística clásica, no menos lo era de las fórmulas estéticas y las técnicas constructivas que se estaban utilizando en la arquitectura del entorno y que confieren al molino un cierto sabor local relacionado con el caserío de la cercana ciudad de San Fernando. Estas fórmulas remiten a los remates que en forma de jarrón coronan los pretiles de las azoteas del edificio, así como a los guardapolvos - de cronología posterior- de los pabellones laterales, de pronunciada forma triangular, que también se localizan en San Fernando relacionados con azoteas y lavaderos.

Dejando a un lado la sólida cantería de piedra ostionera dispuesta en la arcada inferior por obvias razones de resistencia al agua y soporte estático, cuyo uso hidráulico y técnica era habitual en la bahía ${ }^{22}$, el resto del edificio está realizado con hileras de mampostería de la misma piedra, reservándose la cantería para reforzar los vanos y esquinas del inmueble. Este mismo sistema se utiliza con menor calidad constructiva en la crujía lateral en fecha posterior, llegándose a recurrir al ladrillo en los dos últimos arcos laterales años más tarde. La fachada de esta crujía que mira al embalse presenta restos de ventanas y de un arco cegado en la esquina de unión con la crujía mayor, que tal vez correspondiera a algún tipo de mirador de la construcción primitiva que quedó incluido en la ampliación. En la zona inferior de esta crujía aparecen cuatro arcos o canales de desagüe, dos de medio punto y otros dos escarzanos, de los que los dos primeros aún presentan las compuertas basculantes.

La crujía mayor, lugar donde se efectuaba la molienda, aparece articulada en dos cuerpos. El inferior, labrado con sillares, es el que está en contacto con el agua, mostrando en su parte media dos arcos de medio punto de amplia luz que sirven de vías de llenado de la caldera. Desde ellos se despliegan en el lado izquierdo siete arcos cegados de medio punto separados por tajamares apuntados y en lado derecho seis. Sobre los arcos se sitúa una hilera de pequeños huecos rectangulares cuya misión era iluminar el interior de los depósitos de los rodeznos. Separado por una moldura de cierto vuelo, el segundo cuerpo presenta una mayor identidad arquitectónica. Sobre los ojos centrales se abren dos balcones entre pilastras que separan las dos alas de la nave, centralizando la composición, y en línea con los arcos inferiores se abren ventanas. Una ligera cornisa da paso al pretil, también con restos de remates.

En el extremo occidental de esta crujía y con el fin de obtener una visualización simétrica del edificio, se adosó un pabellón de dos plantas con ventanas decoradas con guardapolvos. En torno a él se ha ido generando una serie de construcciones en siglos posteriores que hoy día resultan inaccesibles. A pesar de que en la actualidad no quedan restos de ningún tipo de la primitiva función del molino, sí es clara la ubicación de la sala de molienda cuya disposición en planta alta 
obedece al sistema de aprovechamiento de la energía generada por las mareas. En la planta inferior se localizaba el rodezno, de pala horizontal, que con la fuerza del agua giraba moviendo un eje al que, en la planta superior, se encontraba sujeto la piedra volandera sobre la que se asentaba la tolva, artilugio que suministraba el grano para la molienda ${ }^{23}$. El resto de dependencias que formaban el molino son difícilmente identificables, dado el penoso estado del conjunto, si bien en los años finales del siglo XIX sabemos que constaba de portería, casa habitación, almacén de depósito de grano, sala de molienda, cuadras y pajares. Es decir, una estructura básica que comprendía los elementos esenciales de funcionamiento: vigilancia, almacén, molienda y transporte.

El Molino del Río Arillo dejó de funcionar durante la década de los treinta del presente siglo siendo utili-

Este artículo es fruto de la investigación realizada por los autores con motivo de la propuesta de Inscripción Genérica en el Catálogo General del Patrimonio Histórico de Andalucía del Molino de Marea del Río Arillo (Cádiz) por parte de la Delegación Provincial de Cádiz de la Consejería de Cultura. Han colaborado en el mismo Mercedes Linares Gómez del Pulgar, arquitecta, y los componentes de Octógono, Historiadores del Arte: Juan Arenillas Torrejón, Mercedes Fernández Martín, Juan Carlos Hernández Núñez, Josefa Mata Torres y Luis Martínez Montiel. zado con posterioridad como carpintería y más tarde como almacén de utillaje de pesca y despesca hasta alcanzar el actual crítico nivel de deterioro que en algunas zonas, las menos consistentes, ha llegado al nivel de ruina ${ }^{24}$. El esperado proyecto de rehabilitación del molino como posible equipamiento público del Parque Natural de la Bahía de Cádiz, en el que se encuentra ubicado, puede ser la ocasión idónea para completar la investigación histórica con el estudio arqueológico de sus fábricas. La recuperación de un patrimonio tan singular por su interés etnológico y arquitectónico, modelo casi único de edificaciones preindustriales en la costa gaditana, transciende la puesta en uso de una viejas estructuras y debe estar ligada a la restauración del paisaje del litoral, en el que durante siglos los molinos de mar fueron arquitecturas en los límites de la tierra y el mar.

Con fecha 25 de noviembre el BOJA publica la Resolución del 31 de octubre de 2000 de la DGBC por la que se incoa el procedimiento para la inscripción con carácter genérico en el Catálogo General del Patrimonio Histórico de Andalucía del Molino de Marea del Río Arillo.
I. MENANTEAU, Loic: Desde Bretaña: Una visión general los molinos de marea del litoral atlántico europeo. En Litoral Atlántico 1997. Anuario de Arquitectura y Paisaje. Pág. 108. Por su parte Soledad García Amedeo cita 18 molinos en el entorno geográfico citado. GARCÍA AMEDEO, Soledad: Cádiz y los molinos de Marea. Cádiz. Pág. 19.

2. AZURMENDI, Luis; GÓMEZ, María Angeles: La arquitectura. En Litoral Atlántico 1997. Anuario de Arquitectura y Paisaje. Pág. 29.

3. Sobre una visión general de los molinos de marea, véase MENENTEAU, Loic, Op. Cit.

4. También se le cita como "de San Miguel" o "de los doce apóstoles".

5. RUIZ NIETO-GUERRERO, María del Pilar: Historia urbana de Cádiz. Génesis y formación de una ciudad moderna. Sevilla, 1999. Pág. 233.

6. RUIZ NIETO-GUeRRERO, María del Pilar: Op. Cit. Pág. 2 I5.

7. El terremoto de noviembre de 1755 arruinó buena parte del camino. Desde el año siguiente se inician los proyectos de recuperación que incluían un nuevo trazado muy semejante al actual, abandonándose así el primitivo. En 1756 el ingeniero Silvestre Abarca realiza el primer proyecto en el que se incluye un puente sobre el Río Arillo. CALDERON QUIJANO, J. A. y otros: Cartografía militar y marítima de Cádiz. 15 13-1878. Sevilla, 1978. T.I. Pág. 457. T.Il. Figura 485. También reproducido en MARTINEZ MONTIEL, Luis; BARROS CANEDA, José R.: Más allá de las columnas de Hércules. Cartas, Mapas, Planos y Dibujos desde 1513 a 1823. Cádiz, 2000. Pág. 55.

En 1757 el también ingeniero Enrique Le Gallois de Grimarest realiza otro proyecto que incluye la presencia también de un puente para salvar el Río Arillo. CALDERÓN QUIJANO, J. A. y otros: Op. Cit. T.I Pag. 459-460- T.II Fig 486 y 488.

En cualquier caso parece que hasta 1789 no estaba realizado el nuevo trazado que haciendo curva en Torregorda se adentraba en la ciudad de San Fernando a través de la llamada Calle Real. RUIZ NIETO-GUERRERO, María del Pilar. Op. Cit. Pág. 217.

8. Miguel Álvarez Montañés, aunque se cita como vecino y dedicado al comercio en Cádiz, era natural de Sanlúcar la Mayor en la provincia de Sevilla. Así consta en un poder recíproco para testar otorgado por él y su mujer en Cádiz en 1785. AR- 
CHIVO HISTÓRICO PROVINCIAL DE CÁDIZ (en adelante A.H.P.C.) Protocolos de Cádiz. Leg. 397. I785. s/f

9. A.H.P.C.. Protocolos de Cádiz. Notaría 28. Leg. 5.906. 1798. Fol. I-28v. Citado por CANO REVORA, María Gloria: Cádiz y el Real Cuerpo de Ingenieros Militares ( $697-$ 1 847). Cádiz, 1994. Pág. 344.

10. Resulta de especial interés los argumentos que se exponen en el escrito de solicitud. En él se aprecia la idea que latía en las mentes ilustradas de promocionar las industrias, en este caso transformadoras, que a su vez fueran generadoras de un espacio económico con influencias y beneficios que iban más allá de la propia industria. Tal vez Miguel Alvarez escribió lo que la Junta quería oír pero lo expuesto es reflejo del marco ideológico de la época. A fin de cuentas, trataba de sustentar su industria con la creación de un centro económico que tendría notables repercusiones en su entorno próximo y lejano. En ese sentido, se formaba o se buscaba formar un área de influencia que vendría marcada por la posición en el territorio, el uso que de él se hacía y su repercusión económica y social. Una idea semejante se intentó llevar a cabo unos años antes con otro proyecto relacionado con la conducción de agua potable al Arsenal de La Carraca. En el estudio previo se usan argumentos que remarcan el sentido de áreas de influencia económica que los proyectos llevaban consigo. Al respecto véase BARROS CANEDA, José Ramón: El abastecimiento de aguas al Arsenal de La Carraca. Archivo Hispalense. № 24I. Sevilla, 1996. Pág. 219-231.

1 I. Luis Huet fue trasladado al Campo de Gibraltar y con fecha 27 de marzo de 1798 entregó la plaza de dirección de Ingenieros a José del Pozo y Sucre. CANO REVORA, María Gloria: Op. Cit. Pág. 257.

12. Julián Albo y Helguero en 1792 era Ayudante de Ingeniero y en 1799 pasó a Castilla la Vieja. Fue profesor en la Academia de Alcalá de Henares y en 1804 alcanzó el empleo de Sargento Mayor. CANO REVORA, María Gloria: Op. Cit. Pág. 56-58.

13. En un plano inferior al puente que sorteaba el río, existía otro puente que formaba parte de un pequeño camino secundario.

14. La pesca era una actividad bastante frecuente en los molinos de marea y sobre todo en la zona de la caldera dadas las favorables condiciones que estos espacios generaban al bloquear el flujo del agua. En este caso Miguel Alvarez consigue, pagando un impuesto de 360 reales anuales, evitar que se realicen labores de pesca con redes en el entorno del molino, aunque no consigue evitar mantener una prerrogativa que tenían concedida los ingenieros encargados del arrecife consistente en hacer una despesca una o dos veces al año y repartir el producto entre los trabajadores. Es interesante, al respecto, la narración que recoge Azurmendi sobre esta utilidad añadida de los molinos. En ella cita los acuerdos entre propietarios, molineros y pescadores para explotar esta posibilidad marcándolo como uno de los puntos importantes de relación que el molino establece con su entorno medioambiental. AZURMENDI, Luis; GÓMEZ, Angeles: Op. Cit.
15. Reproducido por MENENTEAU, Loic: Op. Cit. Pág. 107.

16. A.H.P.C.. Protocolos de Cádiz. 1847. Leg. 3.256. Fol. 2.087$2098 \mathrm{v}$.

17. Registro de la Propiedad de Cádiz Número 3. Libro 305. Fol. 191-215.

18. Resulta difícil saber el número de piedras de molienda que tenía el molino ya que han desaparecido. Por cuestiones de simetría constructiva puede pensarse en doce que se corresponderían con los doce arcos menores, quedando el décimo tercero, de mayor luz, como compuerta de desagüe. Avalando esta hipótesis se encuentra Loic Menanteau y el hecho de que al molino también se le conocía con el nombre de los "doce apóstoles" en posible alusión a las doce piedras. En este sentido, Madoz también se refiere a ese número de piedras (MADOZ, Pascual: Diccionario Geográfico Estadístico Histórico de España y sus posesiones de ultramar. Cádiz, 1987. Pág. 58 y 176.

No obstante, y en oposición a esta hipótesis, cabe decir que el arco mayor presenta una configuración semejante a los menores incorporando su hueco de luces y quedando cegado por el lado del mar como los restantes con lo que la función de compuerta de desagüe queda anulada. Además, la anotación del Registro de la Propiedad efectuada en 1889 menciona la "sala de molienda con trece piedras". Registro de la Propiedad de Cádiz. Número 3. Idem.

Por otra parte, en el proyecto inicial siempre se menciona la posibilidad de aumentar el número de piedras en función de la situación, de hecho siempre que se habla del molino se le cita como "de quatro o seis piedras". Esta idea de ampliación fue por tanto una constante desde los primeros momentos $y$ no resulta extraño que finalmente se construyera con las trece piedras modificándose el proyecto inicial.

19. No se ha encontrado ninguna referencia en los repertorios publicados de arquitectos, maestros de obras, alarifes e ingenieros de las ciudades próximas.

20. SERLIO, Sebastiano: Tercero y Cuarto libro de Arquitectura. Ed. Facsímil. Barcelona, 1990. Libro III. Pág. XLI.

2I. SERLIO, Sebastiano: Idem. Libro III. Pág. XLVI.

22. Era común en la zona esta forma de construir, aunque la gran experiencia en edificaciones sobre terrenos cenagosos se dio en la construcción del cercano Arsenal de La Carraca. Al respecto véase BARROS CANEDA, José Ramón: Arquitectura y urbanismo en La Carraca durante el siglo XVIII. Sevilla, 1989.

23. Para un mayor conocimiento de los métodos y formas de la molienda véase ROSA SANTOS, Luis Felipe: Os Moinhos de Mare da Ria Formosa. Faro, 1992. También AZURMENDI PEREZ, Luis: Molinos de mar. Santander, 1985.

24. GARCÍA AMEDEO, Soledad: Op. Cit. 